\title{
Metabolomic analysis revealed that female mussel Mytilus galloprovincialis was sensitive to bisphenol A exposures
}

\author{
ARTICLE · MARCH 2014 \\ DOI: 10.1016/j.etap.2014.02.015 · Source: PubMed
}

CITATIONS

2

4 AUTHORS, INCLUDING:

\section{Chenglong Ji}

Chinese Academy of Sciences

22 PUBLICATIONS 73 CITATIONS

SEE PROFILE
DOWNLOADS

64
VIEWS

77 


\title{
Short communication
}

\section{Metabolomic analysis revealed that female mussel Mytilus galloprovincialis was sensitive to bisphenol A exposures}

\author{
Chenglong $\mathrm{Ji}^{a, b}$, Lei Wei ${ }^{a, b}$, Jianmin Zhao ${ }^{a}$, Huifeng $\mathrm{Wu}^{a, *}$ \\ a Key Laboratory of Coastal Zone Environmental Processes, Yantai Institute of Coastal Zone Research (YIC), Chinese \\ Academy of Sciences (CAS), Shandong Provincial Key Laboratory of Coastal Zone Environmental Processes, YICCAS, \\ Yantai 264003, PR China \\ $\mathrm{b}$ The University of Chinese Academy of Sciences, Beijing 100049, PR China
}

\section{A R T I C L E I N F O}

Article history:

Received 12 December 2013

Received in revised form

17 February 2014

Accepted 20 February 2014

Available online 2 March 2014

Keywords:

Bisphenol A

Mytilus galloprovincialis

Toxicological effects

Metabolomics

\begin{abstract}
A B S T R A C T
Bisphenol A (BPA) is a synthetic compound used in numerous chemicals, such as polycarbonate plastics and epoxy resins, and it can be released into aquatic environment and poses risk on aquatic organisms. In this work, metabolomics was applied to characterize the metabolic responses in mussel Mytilus galloprovincialis exposed to BPA. Our results indicated that the gonad of female mussel was sensitive to BPA exposures ( 1 and $10 \mu \mathrm{g} / \mathrm{L})$ for one month. However, no significant metabolic responses were observed in male mussel gonads exposed to these two concentrations of BPA. Overall, this limited study suggested that the gender differences should be considered in marine ecotoxicology.
\end{abstract}

(c) 2014 Elsevier B.V. All rights reserved.

\section{Introduction}

Bisphenol A (BPA) is a synthetic compound used as a monomer in numerous chemical products, including polycarbonate plastics, epoxy resins and phenoxy resins, which can be utilized in food storage containers and dental sealants (Staples et al., 2000). It is a known endocrine disrupting chemical and is toxic to aquatic organisms (Kang et al., 2007). Due to its wide usage, exposure of aquatic organisms to BPA has increased via the environment and food chains (Kang et al., 2007).

Marine mussel Mytilus galloprovincialis is a good bioindicator used in marine ecotoxicology because of its wide geographic distribution and high tolerance and accumulation of contaminants (Ciacci et al., 2012). Since BPA is a known endocrine disruptor, the gender-specific responses induced by BPA should be considered in ecotoxicology, which may better understand the responsive mechanisms in toxicology

\footnotetext{
* Corresponding author. Tel.: +86 535 2109190; fax: +86 5352109000 .

E-mail address: hfwu@yic.ac.cn (H. Wu).
} 
bioindicators toward BPA exposure. In addition, the gender differences may introduce undesirable variations into induced toxicological effects and distort the interpretation of toxicological mechanisms, when both male and female individuals are used for exposures in same groups.

With the development of system biology, researchers can study a global profile of molecules such as genes, proteins and metabolites and their alterations with high-throughput analyses, which can elucidate the contaminant-induced toxicological effects and mechanisms (Weckwerth, 2011; ji et al., 2013). Among the system biology techniques, metabolomics measures the small molecular weight metabolites involved in all metabolic pathways in biological samples (Wu and Wang, 2010; Liu et al., 2011). The detection of the metabolite changes can provide an insight into the toxicological mechanisms of contaminants in organisms (Williams et al., 2009; Santos et al., 2010; Liu et al., 2011). To our knowledge, no studies attempted to compare the gender-specific responses induced by BPA in marine mussel at metabolite level. In the present study, we used metabolomics to characterize the potential metabolic responses induced by BPA in male and female mussels. Since BPA is an estrogenic endocrine disruptor, the gonad tissue was used in this work.

\section{Materials and methods}

\subsection{Chemicals}

Bisphenol A (BPA, 99\%), dimethyl sulfoxide (DMSO, analytical grade), sodium dihydrogen phosphate $\left(\mathrm{Na}_{2} \mathrm{HPO}_{4}\right.$, analytical grade) and disodium hydrogen phosphate $\left(\mathrm{NaH}_{2} \mathrm{PO}_{4}\right.$, analytical grade) were purchased from Guoyao Chemical Co. Ltd. (Shanghai, China). Extraction solvents, methanol and chloroform (HPLC grade), were purchased from Guoyao Chemical Co. Ltd. (Shanghai, China). Deuterium oxide $\left(\mathrm{D}_{2} \mathrm{O}, 99.9 \%\right.$ in $\left.\mathrm{D}\right)$ and sodium 3-trimethlysilyl [2,2,3,3-D4] propionate (TSP) were purchased from Cambridge Isotope Laboratories (Miami, FL).

\subsection{Animals and experimental design}

Adult mussels M. galloprovincialis (shell length: $5.5-6.0 \mathrm{~cm}$, $n=60$ ) were collected from a pristine site (Yantai, China). After laboratory acclimatization for $7 \mathrm{~d}$, the animals were divided into four groups each containing 15 individuals. The mussels cultured in the normal filtered seawater (FSW) and FSW containing $0.002 \%$ DMSO (v/v) were used as control and solvent control groups, respectively. The other two groups of mussels were exposed to two sublethal concentrations (1 and $10 \mu \mathrm{g} / \mathrm{L}$ ) of BPA, respectively. After exposure for 1 month, all the mussels were immediately dissected for gonad tissues for sex determination and the remaining gonad tissue samples were snap-frozen in liquid nitrogen.

\subsection{Sex determination}

The gonad tissues were carefully fixed in the Bouin's fixative solution after dissection from the mussels. After fixation for $24 \mathrm{~h}$, the gonad tissues were then dehydrated in a progressive series of ethanol and embedded in paraffin. Histological

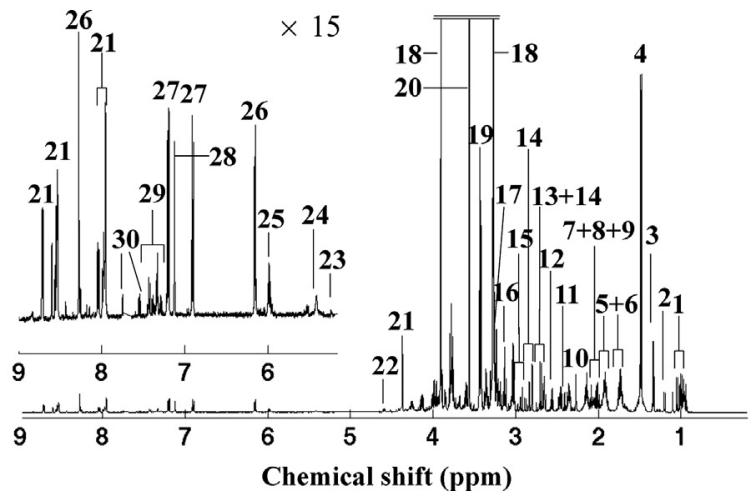

Fig. 1 - A representative 1-dimensional $500 \mathrm{MHz}^{1} \mathrm{H}$ NMR spectrum of gonad tissue extracts of mussel $M$. galloprovincialis from male control group. Keys: (1) branched chain amino acids: valine, leucine and isoleucine, (2) 3-aminoisobutyrate, (3) threonine, (4) alanine, (5) arginine, (6) lysine, (7) glutamate, (8) glutamine, (9) proline, (10) acetoacetate, (11) succinate, (12) $\beta$-alanine, (13) hypotaurine, (14) aspartate, (15) asparagine, (16) malonate, (17) choline, (18) betaine, (19) taurine, (20) glycine, (21) homarine, (22) $\beta$-glucose, (23) $\alpha$-glucose, (24) glycogen, (25) unknown (5.95 ppm), (26) ATP, (27) tyrosine, (28) histidine, (29) phenylalanine and (30) tryptophan.

sections (6- $\mu \mathrm{m}$ thickness) were cut from the paraffin embedded gonad tissues and mounted on slides which were stained with hematoxylin-eosin (HE) and observed under a light microscope (Olympus BX61, Tokyo, Japan).

\subsection{Metabolite extraction}

For each treatment, six individual mussel samples were used for metabolomics analysis. Polar metabolites in gonad tissues were extracted by the modified extraction protocol as described previously (Lin et al., 2007; Wu et al., 2008). Briefly, the gonad tissue (ca. $100 \mathrm{mg}$ ) was homogenized and extracted in $4 \mathrm{~mL} / \mathrm{g}$ of methanol, $5.25 \mathrm{~mL} / \mathrm{g}$ of water and $2 \mathrm{~mL} / \mathrm{g}$ of chloroform. The methanol/water layer with polar metabolites was transferred to a glass vial and dried in a centrifugal concentrator. The tissue extracts were subsequently re-suspended in $600 \mu \mathrm{L}$ phosphate buffer $\left(100 \mathrm{mM} \mathrm{Na}_{2} \mathrm{HPO}_{4}\right.$ and $\mathrm{NaH}_{2} \mathrm{PO}_{4}$, including $0.5 \mathrm{mM}$ TSP, $\mathrm{pH}$ 7.0) in $\mathrm{D}_{2} \mathrm{O}$. The mixture was vortexed and then centrifuged at $3000 \times g$ for $5 \mathrm{~min}$ at $4^{\circ} \mathrm{C}$. The supernatant substance $(550 \mu \mathrm{L})$ was then pipetted into a $5 \mathrm{~mm}$ NMR tube prior to NMR analysis.

\section{5. ${ }^{1} \mathrm{H}$ NMR spectroscopy}

Extracts of gonad tissues were analyzed on a Bruker AV $500 \mathrm{NMR}$ spectrometer performed at $500.18 \mathrm{MHz}$ (at 298 K) as described previously (Zhang et al., 2011). All ${ }^{1} \mathrm{H}$ NMR spectra were phased, baseline-corrected, and calibrated (TSP at $0.0 \mathrm{ppm}$ ) manually using TopSpin (version 2.1, Bruker). Metabolites were identified following the tabulated chemical shifts (Fan, 1996) and using the software, Chenomx (Evaluation Version, Chenomx Inc., Canada). 


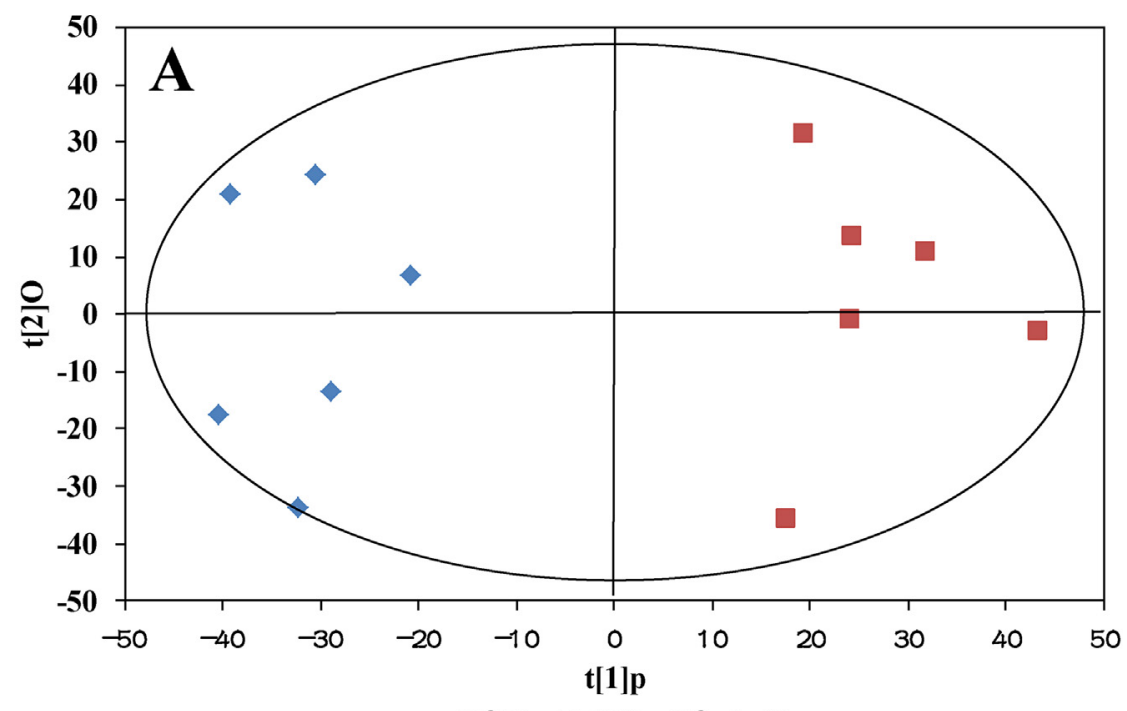

$R^{2} X=46.2 \%, Q^{2}=0.49$

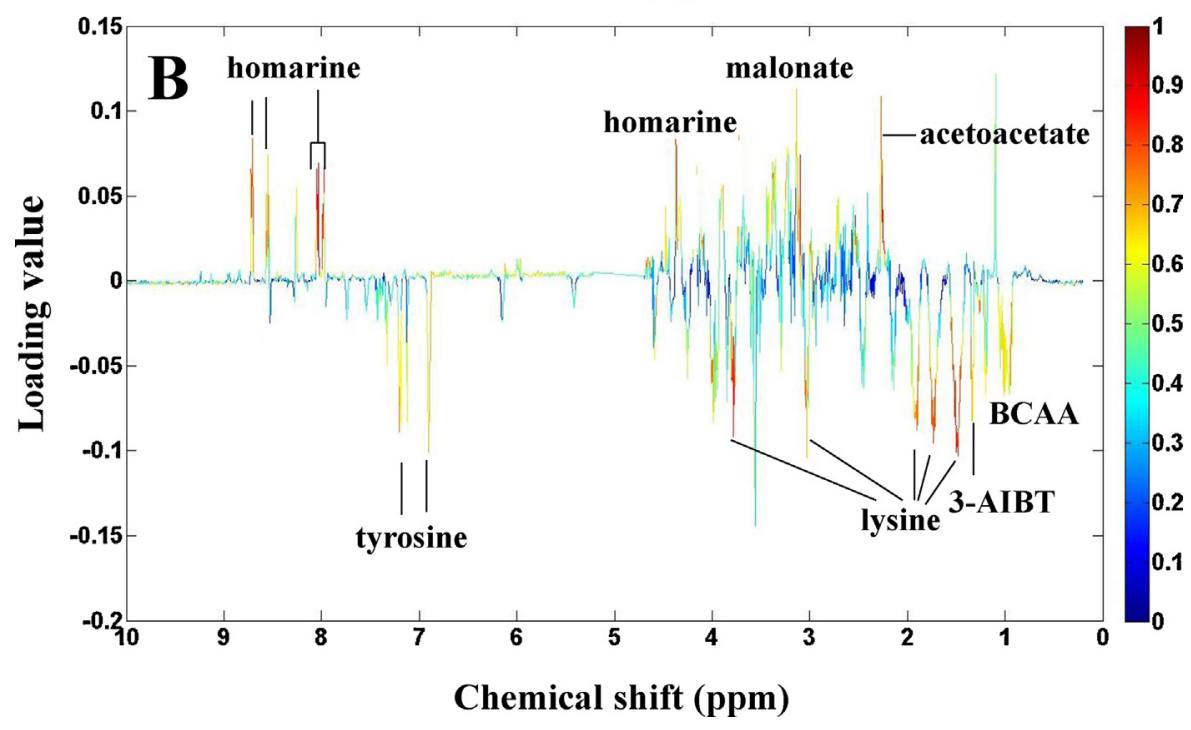

Fig. 2 - O-PLS-DA scores derived from ${ }^{1} \mathrm{H}$ NMR spectra of gonad tissue extracts of $\mathrm{M}$. galloprovincialis from male control $(\diamond)$ and female control $(\square)$ groups (A) and corresponding coefficient plot (B). The color map shows the significance of metabolite variations between the two classes (male control and female control). Peaks in the positive direction indicate metabolites that are more abundant in the female control group. Consequently, metabolites that are more abundant in the male control group are presented as peaks in the negative direction. Abbreviations: BCAA, branched chain amino acids; 3-AIBT, 3-aminoisobutyrate. (For interpretation of the references to color in this figure legend, the reader is referred to the web version of the article.)

\subsection{Spectral pre-processing and multivariate data} analysis

All NMR spectra were processed using custom-written ProMetab software in Matlab (version 7.0; The MathWorks, Natick, MA, USA), as described previously (Parsons et al., 2007; Liu et al., 2011). Multivariate data analysis was performed using the software SIMCA-P ${ }^{+}$(V11.0, Umetric, Sweden). The supervised multivariate data analysis methods, partial least squares discriminant analysis (PLS-DA) and orthogonal projection to latent structure with discriminant analysis (OPLS-DA), were sequentially carried out to uncover and extract the statistically significant metabolite responses induced by BPA exposures. The results were visualized in terms of scores plots to show the classifications and corresponding loadings plots to show the NMR spectral variables contributing to the classifications. The model coefficients were calculated from the coefficients incorporating the weight of the variables in order to enhance interpretability of the model. Then metabolic differences responsible for the classifications between male and female control groups, and between control and BPAexposed groups could be detected in the coefficient-coded loadings plots. The coefficient plots were generated by using MATLAB (V7.0, the Mathworks Inc., Natwick, USA) with an 


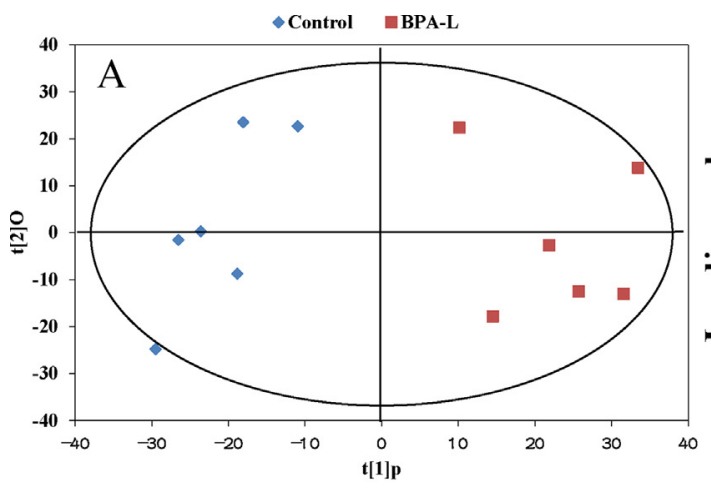

$R^{2} X=40.52 \%, Q^{2}=0.45$

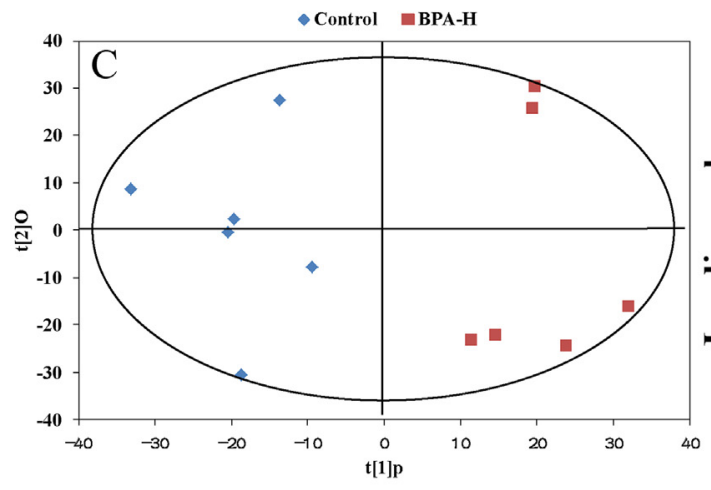

$R^{2} X=41.87 \%, Q^{2}=0.47$

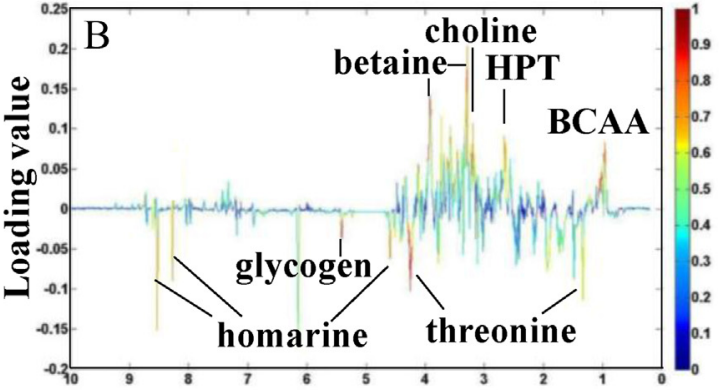

Chemical shift (ppm)

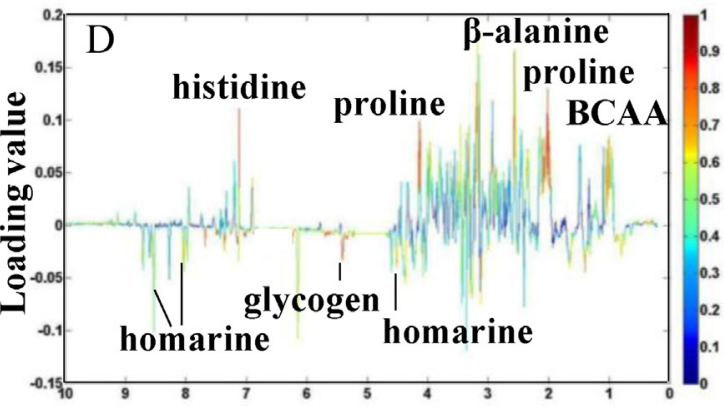

Chemical shift (ppm)

Fig. 3 - O-PLS-DA scores derived from ${ }^{1} \mathrm{H}$ NMR spectra of gonad tissue extracts from solvent control $(\diamond)$ and female mussel groups $(\square$ ) with BPA exposures, (A) $1 \mu \mathrm{g} / \mathrm{L}$ and (C) $10 \mu \mathrm{g} / \mathrm{L}$, and corresponding coefficient plots (B) and (D). The color map shows the significance of metabolite variations between the two classes (solvent control and BPA treatment). Peaks in the positive direction indicate metabolites that are more abundant in $t$ BPA-exposed groups. Consequently, metabolites that are more abundant in the control group are presented as peaks in the negative direction. Abbreviations: BCAA, branched chain amino acids; HPT, hypotaurine. (For interpretation of the references to color in this figure legend, the reader is referred to the web version of the article.)

in-house developed program and were color-coded with absolute value of coefficients ( $r$ ). A hot color (i.e., red) corresponds to the metabolites being highly positive/negative significant in discriminating between groups, while a cool color (i.e. blue) corresponds to no significance. The correlation coefficient was determined according to the test for the significance of the Pearson's product-moment correlation coefficient. The validation of the model was conducted using 6-fold cross validation and the cross-validation parameter $Q^{2}$ was calculated, and an additional validation method, permutation test (permutation number $=200$ ), was also conducted in order to evaluate the validity of the PLS-DA models. The $R^{2}$ in the permutated plot described how well the data fit the derived model, whereas $\mathrm{Q}^{2}$ describes the predictive ability of the derived model and provides a measure of the model quality. If the maximum value of $Q^{2}$ max from the permutation test was smaller than or equal to the $Q^{2}$ of the real model, the model was regarded as a predictable model. Similarly, the $R^{2}$ value and difference between the $R^{2}$ and $Q^{2}$ were used to evaluate the possibility of over-fitted models (Feng et al., 2013).

\section{Results and discussion}

A representative ${ }^{1} \mathrm{H}$ NMR spectrum of gonad tissue extracts from the male control group was shown in Fig. 1. Several classes of metabolites were identified by Chenomx software and labeled in Fig. 1, including amino acids (valine, leucine, isoleucine, alanine, threonine, arginine, lysine, glutamate, glutamine, $\beta$-alanine, aspartate, glycine, tyrosine, phenylalanine, tryptophan and histidine), organic osmolytes (hypotaurine, taurine, homarine and betaine), an intermediate in Krebs cycle (succinate) and energy storage compounds (ATP, glucose and glycogen).

O-PLS-DA resulted in a reliable model for the classification between male control and female control groups, with a $Q^{2}$ value of 0.49 (>0.4) (Fig. 2A). The loading plot (Fig. 2B) displayed the significant metabolic differences between male and female mussel samples from blank groups (seawater control groups). As shown in Fig. 2B, female mussel samples had significantly $(p<0.05)$ higher levels of acetoacetate, malonate and homarine, and lower levels of branched chain amino 
acids (valine, leucine and isoleucine), 3-aminoisobutyrate, lysine and tyrosine than those in male mussel samples. These metabolic differences indicated that there existed intrinsic (gender-specific) biological differences between male and female mussel gonads. These gender differences at the metabolite level could result in gender-specific responses to toxicant exposures. O-PLS-DA indicated that there was no significant metabolic difference between seawater control and solvent control groups from either male or female mussels (data not shown). Therefore, the solvent control groups were used in further metabolomic analysis.

Interestingly, further O-PLS-DA analysis exhibited that both treatments ( 1 and $10 \mu \mathrm{g} / \mathrm{L})$ of BPA did not induce significant metabolic responses in male mussels, with unreliable $Q^{2}$ values $(<0.4)$ (data not shown). In female mussel samples, however, the significant metabolic responses were discovered in both BPA exposures ( 1 and $10 \mu \mathrm{g} / \mathrm{L}$ ) (Fig. 3). Although BPA has been recognized as a weak estrogenic endocrine disruptor for mammals (Kang et al., 2007), our results indicated that the gonad tissue of male mussel $M$. galloprovincialis was not sensitive to BPA exposures at 1 and $10 \mu \mathrm{g} / \mathrm{L}$.

In the female mussel group with the low BPA exposure $(1 \mu \mathrm{g} / \mathrm{L})$, several metabolites were significantly altered including increased branched chain amino acids (valine, leucine and isoleucine), hypotaurine, choline and betaine, and decreased threonine, glycogen and homarine (Fig. 3B). Both hypotaurine and betaine are known organic osmolytes in marine mussels. The elevation and hypotaurine and betaine indicated the osmotic stress induced by BPA $(1 \mu \mathrm{g} / \mathrm{L})$. Betaine is biosynthesized from the oxidation of choline. The elevated choline meant the increased demand of betaine synthesis in female mussel gonad. Homarine is another organic osmolytes in marine mussels, which was decreased to compensate the increases of betaine and hypotaurine in low concentration $(1 \mu \mathrm{g} / \mathrm{L})$ of BPA-treated female mussel samples. In marine mollusks, amino acids are usually involved in both osmotic regulation and cellular energy metabolism (Viant et al., 2003). Since glycogen was decreased, decreased threonine could be related the alteration in energy metabolisms, while branched chain amino acids might be associated with disrupted osmotic regulation in female mussel gonads with the low concentration $(1 \mu \mathrm{g} / \mathrm{L})$ of BPA exposure for one month.

After exposure with the high concentration $(10 \mu \mathrm{g} / \mathrm{L})$ of BPA for one month, some metabolic responses in female mussel gonads were different with those in the low concentration $(1 \mu \mathrm{g} / \mathrm{L})$ of BPA, except branched chain amino acids, homarine and glycogen (Fig. 3D). Both betaine and hypotaurine were not significantly elevated in female mussel samples with BPA exposure at the high concentration $(10 \mu \mathrm{g} / \mathrm{L})$. Interestingly, several amino acids including proline, $\beta$-alanine and histidine were significantly elevated, instead of the elevated organic osmolytes, betaine and hypotaurine in the low concentration of BPA-treated group. As mentioned above, the elevated amino acids probably meant the osmotic stress. However, these differential metabolic responses clearly indicated the different responsive mechanisms related to osmotic regulation to these two BPA exposures in female mussel gonads.

In summary, our results indicated that the gonad of female mussel was sensitive to BPA exposures (1 and $10 \mu \mathrm{g} / \mathrm{L}$ ) for one month. No significant metabolic responses were observed in male mussel gonads exposed to these two concentrations of BPA. Overall, both concentrations of BPA induced osmotic stress and disruption in energy metabolism. In particular, the mussels with $1 \mu \mathrm{g} / \mathrm{L}$ of BPA exposure increased betaine and hypotaurine to respond to the osmotic stress, while those with $10 \mu \mathrm{g} / \mathrm{L}$ of BPA exposure mobilized more amino acids to cope with osmotic stress. This limited study suggested the gender differences should be considered in marine ecotoxicology.

\section{Conflict of interest statement}

The authors declare that there are no conflicts of interest.

\section{Transparency document}

The Transparency document associated with this article can be found in the online version.

\section{Acknowledgments}

This work was supported by The 100 Talents Program of the Chinese Academy of Sciences and Key Deployment Program of Chinese Academy of Sciences (KZZD-EW-14-03).

\section{REFERENCES}

Ciacci, C., Barmo, C., Gallo, G., Maisano, M., Cappello, T., D’Agata, A., Leonzio, C., Mauceri, A., Fasulo, S., Canesi, L., 2012. Effects of sublethal, environmentally relevant concentrations of hexavalent chromium in the gills of Mytilus galloprovincialis. Aquat. Toxicol. 120-121, 109-118.

Fan, W.M.T., 1996. Metabolite profiling by one- and two-dimensional NMR analysis of complex mixtures. Prog. Nucl. Magn. Reson. 28, 161-219.

Feng, J., Li, J., Wu, H., Chen, Z., 2013. Metabolic responses of HeLa cells to silica nanoparticles by NMR-based metabolomic analyses. Metabolomics 13, 874-886.

Ji, C., Wu, H., Wei, L., Zhao, J., Yu, J., 2013. Proteomic and metabolomic analysis reveal gender-specific responses of mussel Mytilus galloprovincialis to 2,2',4,4'-tetrabromodiphenyl ether (BDE 47). Aquat. Toxicol. 140-141, 449-457.

Kang, J.-H., Aasi, D., Katayama, Y., 2007. Bisphenol A in the aquatic environment and its endocrine-disruptive effects on aquatic organisms. Crit. Rev. Toxicol. 37, 607-625.

Lin, C.Y., Wu, H., Tjeerdema, R.S., Viant, M.R., 2007. Evaluation of metabolite extraction strategies from tissue samples using NMR metabolomics. Metabolomics 3, 55-67.

Liu, X., Zhang, L., You, L., Cong, M., Zhao, J., Wu, H., Li, C., Liu, D., Yu, J., 2011. Toxicological responses to acute mercury exposure for three species of Manila clam Ruditapes philippinarum by NMR-based metabolomics. Environ. Toxicol. Pharmacol. 31, 323-332.

Parsons, H.M., Ludwig, C., Gunther, U.L., Viant, M., 2007. Improved classification accuracy in 1- and 2-dimensional NMR metabolomics data using the variance stabilising generalised logarithm transformation. BMC Bioinform. 8, 234.

Santos, E.M., Ball, J.S., Williams, T.D., Wu, H., Ortega, F., van Aerle, R., Katsiadaki, I., Falciani, F., Viant, M.R., Chipman, J.K., Tyler, C.R., 2010. Identifying health impacts of exposure to copper 
using transcriptomics and metabolomics in a fish model. Environ. Sci. Technol. 44, 820-826.

Staples, C.A., Dorn, P.B., Klecka, G.M., O’Block, S.T., Branson, D.R., Harris, L.R., 2000. Bisphenol A concentrations in receiving waters near US manufacturing and processing facilities. Chemosphere 40, 521-525.

Viant, M.R., Rosenblum, E.S., Tjeerdema, R.S., 2003. NMR-based metabolomics: a powerful approach for characterizing the effects of environmental stressors on organism health. Environ. Sci. Technol. 37, 4982-4989.

Weckwerth, W., 2011. Green systems biology - from single genomes, proteomes and metabolomes to ecosystems research and biotechnology. J. Proteomics 75, 284-305.

Williams, T.D., Wu, H., Santos, E.M., Ball, J., Katsiadaki, I., Brown, M.M., Baker, P., Ortega, F., Falciani, F., Craft, J.A., Tyler, C.R., Chipman, J.K., Viant, M.R., 2009.
Hepatic transcriptomic and metabolomic responses in the stickleback (Gasterosteus aculeatus) exposed to environmentally relevant concentrations of dibenzanthracene. Environ. Sci. Technol. 43, 6341-6348.

Wu, H., Southam, A.D., Hines, A., Viant, M.R., 2008. High throughput tissue extraction protocol for NMR and mass spectrometry based metabolomics. Anal. Biochem. 372, 204-212.

Wu, H., Wang, W.-X., 2010. NMR-based metabolomic studies on the toxicological effects of cadmium and copper on green mussels Perna viridis. Aquat. Toxicol. 100, 339-345.

Zhang, L., Liu, X., You, L., Zhou, D., Wu, H., Li, L., Zhao, J., Feng, J., Yu, J., 2011. Metabolic responses in gills of Manila clam Ruditapes philippinarum exposed to copper using NMR-based metabolomics. Mar. Environ. Res. 72, 33-39. 